\title{
On poisson-amarendra distribution and its applications
}

\begin{abstract}
In this paper a simple method for obtaining moments of Poisson-Amarendra distribution (PAD) introduced by Shanker ${ }^{1}$ has been suggested and hence the first four moments about origin and the variance has been given. The applications and the goodness of fit of the PAD have been discussed with data-sets relating to ecology, genetics and thunderstorms and the fit is compared with Poisson distribution, PoissonLindley distribution (PLD) introduced by Sankaran ${ }^{2}$ and Poisson-Sujatha distribution introduced by Shanker ${ }^{3}$ and the fit of PAD shows satisfactory fit in most of data-sets.

Keywords: amarendra distribution poisson-amarendra distribution, poisson-lindley distribution, poisson-sujatha distribution, moments, estimation of parameter, goodness of fit
\end{abstract}

Volume 4 Issue 3 - 2016

\section{Rama Shanker,' Hagos Fesshaye ${ }^{2}$}

'Department of Statistics, Eritrea Institute of Technology, Eritrea ${ }^{2}$ Department of Economics, College of Business and Economics, Eritrea

Correspondence: Rama Shanker, Department of Statistics, Eritrea Institute of Technology, Asmara, Eritrea, Email shankerrama2009@gmail.com

Received: July 19, 2016 | Published: August 27, 2016

\section{Introduction}

The Poisson-Amarendra distribution (PAD) defined by its probability mass function (p.m.f.)

$$
\begin{gathered}
P(X=x)=\frac{\theta^{4}}{\theta^{3}+\theta^{2}+2 \theta+6} \frac{x^{3}+(\theta+7) x^{2}+\left(\theta^{2}+5 \theta+15\right) x+\left(\theta^{3}+4 \theta^{2}+7 \theta+10\right)}{(\theta+1)^{x+4}} \\
; x=0,1,2, \ldots, \theta>0
\end{gathered}
$$

has been introduced by Shanker ${ }^{1}$ for modeling count data-sets. Shanker ${ }^{1}$ has shown that PAD is a Poisson mixture of Amarendra distribution introduced by Shanker ${ }^{4}$ when the parameter $\lambda$ of Poisson distribution follows Amarendra distribution having probability density function (p.d.f.)

$$
f(\lambda ; \theta)=\frac{\theta^{4}}{\theta^{3}+\theta^{2}+2 \theta+6}\left(1+\lambda+\lambda^{2}+\lambda^{3}\right) e^{-\theta \lambda} ; \lambda>0, \theta>0
$$

We have

$$
\begin{gathered}
P(X=x)=\int_{0}^{\infty} \frac{e^{-\lambda} \lambda^{x}}{x !} \cdot \frac{\theta^{4}}{\theta^{3}+\theta^{2}+2 \theta+6}\left(1+\lambda+\lambda^{2}+\lambda^{3}\right) e^{-\theta \lambda} d \lambda \\
=\frac{\theta^{4}}{\left(\theta^{3}+\theta^{2}+2 \theta+6\right) x !} \int_{0}^{\infty} \lambda^{x}\left(1+\lambda+\lambda^{2}+\lambda^{3}\right) e^{-(\theta+1) \lambda} d \lambda \\
=\frac{\theta^{4}}{\theta^{3}+\theta^{2}+2 \theta+6} \cdot \frac{x^{3}+(\theta+7) x^{2}+\left(\theta^{2}+5 \theta+15\right) x+\left(\theta^{3}+4 \theta^{2}+7 \theta+10\right)}{(\theta+1)^{x+4}}
\end{gathered}
$$

$$
; x=0,1,2, \ldots, \theta>0
$$

which is the Poisson-Amarendra distribution (PAD).

It has been shown by Shanker ${ }^{4}$ that Amarendra distribution is a four component mixture of exponential $(\theta)$ distribution, a gamma
$(2, \theta)$ distribution, a gamma $(3, \theta)$ distribution and a gamma $(4, \theta)$ distribution with their mixing proportions, $\frac{\theta^{3}}{\theta^{3}+\theta^{2}+2 \theta+6}, \frac{\theta^{2}}{\theta^{3}+\theta^{2}+2 \theta+6}$ , $\frac{2 \theta}{\theta^{3}+\theta^{2}+2 \theta+6}$ and $\frac{6}{\theta^{3}+\theta^{2}+2 \theta+6}$ respectively. Shanker ${ }^{4}$ has discussed its various mathematical and statistical properties including its shape for different values of its parameter, moment generating function, moments, skewness, kurtosis, hazard rate function, mean residual life function, stochastic orderings, mean deviations, Bonferroni and Lorenz curves, amongst others. Further, Shanker ${ }^{4}$ has also discussed the estimation of its parameter using maximum likelihood estimation and method of moments along with applications for modeling lifetime data and observed that it gives much closer fit than Akash, Shanker and Sujatha distributions introduced by Shanker, ${ }^{5-7}$ Lindley $^{8}$ and exponential distributions. It would be worth mentioning that Shanker ${ }^{5-7}$ has proposed Akash, Shanker and Sujatha, distributions along with their various mathematical and statistical properties to model lifetime data arising from engineering and biomedical sciences and showed that these distributions provide much closer fit than Lindley and exponential distributions.

The Poisson-Lindley distribution (PLD) defined by its p.m.f.

$$
P(X=x)=\frac{\theta^{2}(x+\theta+2)}{(\theta+1)^{x+3}} ; x=0,1,2, \ldots, \quad \theta>0 .
$$

has been introduced by Sankaran [2] to model count data. The PLD is a Poisson mixture of Lindley [8] distribution when the parameter $\lambda$ of Poisson distribution follows Lindley [8] distribution with its p.d.f.

$$
f(\lambda, \theta)=\frac{\theta^{2}}{\theta+1}(1+\lambda) e^{-\theta \lambda} ; \quad x>0, \theta>0
$$

Shanker \& Hagos ${ }^{9}$ has done detailed study about applications of Poisson-Lindley distribution for modeling count data from biological sciences and showed that it gives better fit than Poisson-distribution. Shanker et al., ${ }^{10}$ discussed the comparative study of zero-truncated Poisson and Poisson-Lindley distributions and observed that in majority of data sets zero-truncated Poisson-Lindley distribution gives better fit. 
Shanker ${ }^{11}$ obtained Poisson-Sujatha distribution (PSD) having p.m.f.

$$
P(X=x)=\frac{\theta^{3}}{\theta^{2}+\theta+2} \cdot \frac{x^{2}+(\theta+4) x+\left(\theta^{2}+3 \theta+4\right)}{(\theta+1)^{x+3}} ; x=0,1,2, \ldots, \theta>0
$$

by compounding Poisson distribution with Sujatha distribution, introduced by Shanker ${ }^{7}$ having p.d.f.

$$
f(x ; \theta)=\frac{\theta^{3}}{\theta^{2}+\theta+2}\left(1+x+x^{2}\right) e^{-\theta x} ; x>0, \theta>0
$$

Sujatha distribution introduced by Shanker ${ }^{7}$ is a better model than exponential and Lindley distributions for modeling lifetime data from biomedical science and engineering. Further, Shanker \& Hagos $^{11}$ has detailed study about applications of Poisson-Sujatha distribution (PSD) for modeling count data from biological science and observed that it gives better fit than Poisson-Lindley (PLD) and Poisson-distribution. Shanker \& Hagos ${ }^{12,13}$ have obtained the sizebiased Poisson-Sujatha distribution (SBPSD) and zero-truncated Poisson-Sujatha distribution (ZTPSD) and discussed their various mathematical and statistical properties, estimation of their parameter and applications. Further, Shanker \& Hagos ${ }^{14}$ have detailed study regarding applications of zero-truncated Poisson (ZTPD), zerotruncated Poisson-Lindley distribution (ZTPLD), and zero-truncated Poisson-Sujatha distribution (ZTPSD) for modeling data-sets excluding zero counts from demography and biological sciences and concluded that in majority of data-sets ZTPSD gives better fit than ZTPD and ZTPLD.

In this paper a simple method for obtaining moments of PoissonAmarendra distribution (PAD) introduced by Shanker ${ }^{1}$ has been suggested and hence the first four moments about origin and the variance has been presented. It seems that not much work has been done on the applications of PAD so far. The PAD has been fitted to some data-sets relating to ecology, genetics and thunderstorms and its goodness of fit has been compared with Poisson distribution (PD), Poisson-Lindley distribution (PLD), Poisson-Sujatha distribution (PSD).

\section{Moments of pad}

Using (1.3), the $r^{\text {th }}$ moment about origin of PAD (1.1) can be obtained as

$$
\mu_{r}^{\prime}=E\left[E\left(X^{r} \mid \lambda\right)\right]=\frac{\theta^{4}}{\theta^{3}+\theta^{2}+2 \theta+6} \int_{0}^{\infty}\left[\sum_{x=0}^{\infty} x^{r} \frac{e^{-\lambda} \lambda^{x}}{x !}\right]\left(1+\lambda+\lambda^{2}+\lambda^{3}\right) e^{-\theta \lambda} d \lambda
$$

clearly that the expression under the bracket in (2.1) is the $r^{\text {th }}$ moment about origin of the Poisson distribution. Taking $r=1$ in (2.1) and using the mean of the Poisson distribution, the mean of the PAD (1.1) can be obtained as

$$
\mu_{1}^{\prime}=\frac{\theta^{4}}{\theta^{3}+\theta^{2}+2 \theta+6} \int_{0}^{\infty} \lambda\left(1+\lambda+\lambda^{2}+\lambda^{3}\right) e^{-\theta \lambda} d \lambda=\frac{\theta^{3}+2 \theta^{2}+6 \theta+24}{\theta\left(\theta^{3}+\theta^{2}+2 \theta+6\right)}
$$

Again taking $r=2$ in (2.1) and using the second moment about origin of the Poisson distribution, the second moment about origin of the PAD (1.1) can be obtained as

$$
\mu_{2}^{\prime}=\frac{\theta^{4}}{\theta^{3}+\theta^{2}+2 \theta+6} \int_{0}^{\infty}\left(\lambda^{2}+\lambda\right)\left(1+\lambda+\lambda^{2}+\lambda^{3}\right) e^{-\theta \lambda} d \lambda=\frac{\theta^{4}+4 \theta^{3}+12 \theta^{2}+48 \theta+120}{\theta^{2}\left(\theta^{3}+\theta^{2}+2 \theta+6\right)}
$$

Similarly, taking $r=3$ and 4 in (2.1) and using the third and fourth moments about origin of the Poisson distribution, the third and the fourth moments about origin of the PAD (1.1) are obtained as

$$
\begin{gathered}
\mu_{3}^{\prime}=\frac{\theta^{5}+8 \theta^{4}+30 \theta^{3}+120 \theta^{2}+480 \theta+720}{\theta^{3}\left(\theta^{3}+\theta^{2}+2 \theta+6\right)} \\
\mu_{4}^{\prime}=\frac{\theta^{6}+16 \theta^{5}+84 \theta^{4}+360 \theta^{3}+1680 \theta^{2}+5040 \theta+5040}{\theta^{4}\left(\theta^{3}+\theta^{2}+2 \theta+6\right)}
\end{gathered}
$$

The variance of PAD (1.1) can thus be obtained as

$$
\mu_{2}=\frac{\theta^{7}+4 \theta^{6}+14 \theta^{5}+58 \theta^{4}+144 \theta^{3}+156 \theta^{2}+240 \theta+144}{\theta^{2}\left(\theta^{3}+\theta^{2}+2 \theta+6\right)^{2}}
$$

\section{Parameter estimation}

Maximum likelihood estimate (MLE) of the parameter: Let $\left(x_{1}, x_{2}, \ldots, x_{n}\right)$ be a random sample of size $n$ from the PAD (1.1) and let $f_{x}$ be the observed frequency in the sample corresponding to $X=x(x=1,2,3, \ldots, k)$ such that $\sum^{k} f_{x}=n$, where $k$ is the largest observed value having non-zero frequency. The likelihood function $L$ of the PAD (1.1) is given by

$$
\left.L=\left(\frac{\theta^{4}}{\theta^{3}+\theta^{2}+2 \theta+6}\right)^{n} \frac{1}{(\theta+1)_{x=1}^{k} f_{x}(x+4)} \prod_{x=1}^{k}\left[+\left(\theta^{3}+4 \theta^{2}+7 \theta+10\right)\right] x^{3}+(\theta+7) x^{2}+\left(\theta^{2}+5 \theta+15\right) x\right]^{f_{x}}
$$

The log likelihood function is thus obtained as

$$
\log L=n \log \left(\frac{\theta^{4}}{\theta^{3}+\theta^{2}+2 \theta+6}\right)-\sum_{x=1}^{k} f_{x}(x+4) \log (\theta+1)+\sum_{x=1}^{k} f_{x} \log \left[x^{3}+(\theta+7) x^{2}+\left(\theta^{2}+5 \theta+15\right) x+\left(\theta^{3}+4 \theta^{2}+7 \theta+10\right)\right]
$$

The first derivative of the log likelihood function is given by

$$
\frac{d \log L}{d \theta}=\frac{4 n}{\theta}-\frac{n\left(3 \theta^{2}+2 \theta+2\right)}{\left(\theta^{3}+\theta^{2}+2 \theta+6\right)}-\frac{n(\bar{x}+4)}{\theta+1}+\sum_{x=1}^{k} \frac{\left[x^{2}+(2 \theta+5) x+\left(3 \theta^{2}+8 \theta+7\right)\right] f_{x}}{\left[x^{3}+(\theta+7) x^{2}+\left(\theta^{2}+5 \theta+15\right) x+\left(\theta^{3}+4 \theta^{2}+7 \theta+10\right)\right]}
$$


where $\bar{x}$ is the sample mean.

The maximum likelihood estimate (MLE), $\hat{\theta}$ of $\theta$ of PAD (1.1) is the solution of $\frac{d \log L}{d \theta}=0$ and is the solution of the following non-
linear equation

$$
\frac{4 n}{\theta}-\frac{n\left(3 \theta^{2}+2 \theta+2\right)}{\left(\theta^{3}+\theta^{2}+2 \theta+6\right)}-\frac{n(\bar{x}+4)}{\theta+1}+\sum_{x=1}^{k} \frac{\left[x^{2}+(2 \theta+5) x+\left(3 \theta^{2}+8 \theta+7\right)\right] f_{x}}{\left[x^{3}+(\theta+7) x^{2}+\left(\theta^{2}+5 \theta+15\right) x+\left(\theta^{3}+4 \theta^{2}+7 \theta+10\right)\right]}=0
$$

This non-linear equation can be solved using any numerical iteration methods such as Newton- Raphson method, Bisection method, Regula -Falsi method etc. In this paper, Newton-Raphson method has been used to solve the above equation for estimating the parameter $\theta$.

Method of moment estimate (MOME) of the parameter: Let $\left(x_{1}, x_{2}, \ldots, x_{n}\right)$ be a random sample of size $n$ from the PAD (1.1). Equating the population mean to the corresponding sample mean, the MOME $\tilde{\theta}$ of $\theta$ of PAD (1.1) is the solution of the following biquadratic equation

$$
\bar{x} \theta^{4}+(\bar{x}-1) \theta^{3}+2(\bar{x}-1) \theta^{2}+6(\bar{x}-1) \theta-24=0
$$

where $\bar{x}$ is the sample mean.

\section{Goodness of fit and applications}

The Poisson distribution is a suitable statistical model for the situations where events are independent and mean equals variance, which is unrealistic in most of data sets in biological science and thunderstorms. Further, the negative binomial distribution is a possible alternative to the Poisson distribution when successive events are possibly dependent Johnson et al., ${ }^{15}$ but for fitting negative binomial distribution (NBD) to the count data, mean must be less than the variance. In biological science and thunderstorms, these conditions are not fully satisfied. Generally, the count data in biological science and thunderstorms are either over-dispersed or under-dispersed. The main reason for selecting PAD, PSD, and PLD to fit data from biological science and thunderstorms are that these distributions are always over-dispersed and PAD has some flexibility over PSD and PLD.

\section{Applications in ecology}

Ecology is the branch of biology which deals with the relations and interactions between organisms and their environment, including their organisms. The organisms and their environment in the nature are complex, dynamic, interdependent, mutually reactive and interrelated. Ecology deals with various principles which govern such relationship between organisms and their environment. Fisher et al., ${ }^{16}$ firstly discussed the applications of Logarithmic series distribution (LSD) to model count data in ecology. Later, Kempton ${ }^{17}$ fitted the generalized form of Fisher's Logarithmic series distribution (LSD) to model insect data and concluded that it gives a superior fit as compared to ordinary Logarithmic series distribution (LSD). He also concluded that it gives better explanation for the data having exceptionally long tail. Tripathi \& Gupta $^{18}$ proposed another generalization of the Logarithmic series distribution (LSD) which is flexible to describe short-tailed as well as long-tailed data and fitted it to insect data and found that it gives better fit as compared to ordinary Logarithmic series distribution. Mishra $\&$ Shanker ${ }^{19}$ have discussed applications of generalized logarithmic series distributions (GLSD) to models data in ecology. Shanker \& Hagos ${ }^{10}$ have tried to fit PLD for data relating to ecology and observed that PLD gives satisfactory fit. Shanker \& Hagos $^{11}$ has discussed applications of PSD to model count data from biological science and concluded that PSD gives superior fit than PLD in majority of data.

In this section an attempt has been made to fit Poisson distribution (PD), Poisson -Lindley distribution (PLD), Poisson-Sujatha distribution (PSD) and Poisson-Amarendra distribution (PAD) to many count data from biological sciences using maximum likelihood estimates. The data were on haemocytometer yeast cell counts per square, on European red mites on apple leaves and European corn borers per plant.

It is obvious from above tables that in Table 1, PD gives better fit than PLD, PSD and PAD; in Table 2 PAD gives better fit than PD, PLD, and PSD while in Table 3, PSD gives better fit than PD, PLD and PAD.

\begin{tabular}{|c|c|c|c|c|c|}
\hline \multirow{2}{*}{$\begin{array}{l}\text { Number of yeast } \\
\text { cells per square }\end{array}$} & \multirow{2}{*}{$\begin{array}{l}\text { Observed } \\
\text { frequency }\end{array}$} & \multicolumn{4}{|c|}{ Expected frequency } \\
\hline & & PD & PLD & PSD & PAD \\
\hline 0 & 213 & 202.1 & 234.0 & 233.7 & 233.7 \\
\hline I & 128 & 138.0 & 99.4 & 99.6 & 98.4 \\
\hline 2 & 37 & 47.1 & 40.5 & 41.0 & 41.0 \\
\hline 3 & 18 & $10.7)$ & 16.0 & 16.3 & $16.7)$ \\
\hline 4 & 3 & 1.8 & 6.2 & 6.7 & 6.5 \\
\hline 5 & 1 & 0.2 & 2.4 & 2.3 & 2.4 \\
\hline 6 & 0 & 0.1 & 1.5 & 0.9 & 1.3 \\
\hline Total & & 400.0 & 400.0 & 400.0 & 400.0 \\
\hline
\end{tabular}

Table I Observed and expected number of Haemocytometer yeast cell counts per square observed by 'Student' 1907 


\begin{tabular}{|c|c|c|c|c|c|}
\hline \multirow{2}{*}{$\begin{array}{l}\text { Number of yeast } \\
\text { cells per square }\end{array}$} & \multirow{2}{*}{$\begin{array}{l}\text { Observed } \\
\text { frequency }\end{array}$} & \multicolumn{4}{|c|}{ Expected frequency } \\
\hline & & PD & PLD & PSD & PAD \\
\hline ML Estimate & & $\hat{\theta}=0.6825$ & $\hat{\theta}=1.950236$ & $\hat{\theta}=2.373052$ & $\hat{\theta}=2.759978$ \\
\hline$\chi^{2}$ & & 10.08 & 11.04 & 10.86 & 12.01 \\
\hline d.f. & & 2 & 2 & 2 & 2 \\
\hline $\mathrm{p}$-value & & 0.0065 & 0.004 & 0.0044 & 0.0025 \\
\hline
\end{tabular}

Table 2 Observed and expected number of red mites on Apple leaves

\begin{tabular}{|c|c|c|c|c|c|}
\hline \multirow{2}{*}{$\begin{array}{l}\text { Number Mites Per } \\
\text { leaf }\end{array}$} & \multirow{2}{*}{$\begin{array}{l}\text { Observed } \\
\text { frequency }\end{array}$} & \multicolumn{4}{|c|}{ Expected frequency } \\
\hline & & PD & PLD & PSD & PAD \\
\hline 0 & 38 & 25.3 & 35.8 & 35.3 & 35.3 \\
\hline 1 & 17 & 29.1 & 20.7 & 20.9 & 20.8 \\
\hline 2 & 10 & 16.7 & 11.4 & 11.6 & 11.7 \\
\hline 3 & 9 & 6.4 & 6.0 & 6.1 & 6.2 \\
\hline 4 & 3 & $\left.\begin{array}{l}1.8 \\
0.4\end{array}\right\}$ & $\left.\begin{array}{l}3.1 \\
1.6\end{array}\right)$ & $\begin{array}{l}3.1 \\
1.5\end{array}$ & $\left.\begin{array}{l}3.1 \\
1.5\end{array}\right\}$ \\
\hline 5 & 2 & 0.2 & $0.8\}$ & $0.7\}$ & 0.8 \\
\hline $\begin{array}{l}6 \\
7+\end{array}$ & $\begin{array}{l}1 \\
0\end{array}$ & 0.1 & 0.6 & 0.8 & 0.6 \\
\hline Total & 80 & 80.0 & 80.0 & 80.0 & 80.0 \\
\hline ML estimate & & $\hat{\theta}=1.15$ & $\hat{\theta}=1.255891$ & $\hat{\theta}=1.264683$ & $\hat{\theta}=2.04047$ \\
\hline$\chi^{2}$ & & 18.27 & 2.47 & 2.52 & 2.41 \\
\hline d.f. & & 2 & 3 & 3 & 3 \\
\hline $\mathrm{p}$-value & & 0.0001 & 0.4807 & 0.4719 & 0.4918 \\
\hline
\end{tabular}

Table 3 Observed and expected number of European corn- borer of Mc. Guire et al., ${ }^{23}$

\begin{tabular}{|c|c|c|c|c|c|}
\hline \multirow{2}{*}{$\begin{array}{l}\text { Number of bores } \\
\text { per plant }\end{array}$} & \multirow{2}{*}{$\begin{array}{l}\text { Observed } \\
\text { frequency }\end{array}$} & \multicolumn{4}{|c|}{ Expected frequency } \\
\hline & & PD & PLD & PSD & PAD \\
\hline 0 & 188 & 169.4 & 194.0 & 193.6 & 194.2 \\
\hline I & 83 & 109.8 & 79.5 & 79.6 & 78.6 \\
\hline 2 & 36 & 35.6 & 31.3 & 31.6 & 31.6 \\
\hline 3 & 14 & 7.8 & $12.0)$ & $12.1)$ & 12.3 \\
\hline 4 & 2 & $1.2\}$ & 4.5 & 4.5 & 4.7 \\
\hline 5 & 1 & 0.2 & 2.7 & 2.6 & 2.6 \\
\hline Total & 324 & 324.0 & 324.0 & 324.0 & 324.0 \\
\hline ML estimate & & $\hat{\theta}=0.648148$ & $\hat{\theta}=2.043252$ & $\hat{\theta}=2.858180$ & $\hat{\theta}=2.858180$ \\
\hline$\chi^{2}$ & & 15.19 & 1.29 & 1.16 & 1.4 \\
\hline d.f. & & 2 & 2 & 2 & 2 \\
\hline $\mathrm{p}$-value & & 0.0005 & 0.5247 & 0.5599 & 0.4966 \\
\hline
\end{tabular}




\section{Applications in genetics}

Genetics is the branch of biological science which deals with heredity and variation. Heredity includes those traits or characteristics which are transmitted from generation to generation, and is therefore fixed for a particular individual. Variation, on the other hand, is mainly of two types, namely hereditary and environmental. Hereditary variation refers to differences in inherited traits whereas environmental variations are those which are mainly due to environment. Much quantitative works seem to be done to model count data in genetics but so far no works has been done on fitting of PAD to data in genetics. The segregation of chromosomes has been studied using statistical tool, mainly chi-square $\left(\chi^{2}\right)$. In the analysis of data observed on chemically induced chromosome aberrations in cultures of human leukocytes, Loeschke \& Kohler $^{20}$ suggested the negative binomial distribution while Janardan \& Schaeffer ${ }^{21}$ suggested modified Poisson distribution. Mishra \& Shanker ${ }^{19}$ have discussed applications of generalized Logarithmic series distributions (GLSD) to model data in mortality, ecology and genetics. Shanker \& $\operatorname{Hagos}^{9}$ have detailed study on the applications of PLD to model data from genetics. Shanker \& Hagos ${ }^{11}$ has detailed study on modeling of count data in genetics using PSD. In this section an attempt has been made to fit to PAD, PSD, PLD and PD to data from genetics using maximum likelihood estimate. Also an attempt has been made to fit PAD, PSD, PLD, and PD to the data of Catcheside et al., ${ }^{22}$ in Table 5-7.
It is obvious that in Table $4 \& 7$, PLD gives better fit than PD, PSD and PAD; in Table $5 \& 6$, PAD gives better fit than PD, PLD, and PSD.

\section{Applications in thunderstorms}

In thunderstorm activity, the occurrence of successive thunderstorm events (THE's) is generally a dependent process meaning that the occurrence of a THE indicates that the atmosphere is unstable and the conditions are favorable for the formation for further thunderstorm activity. The negative binomial distribution (NBD) is a possible alternative to the Poisson distribution when successive events are possibly dependent Johnson et al., ${ }^{15}$ The theoretical and empirical justification for using the NBD to describe THE activity has been fully explained and discussed by Falls et al. ${ }^{24}$ Further, for fitting Poisson distribution to the count data equality of mean and variance must be satisfied. Similarly, for fitting NBD to the count data, mean must be less than the variance. In THE, these conditions are not fully satisfied. As a model to describe the frequencies of thunderstorms (TH's), given an occurrence of THE, the PAD can be considered over PSD, PLD and PD because PAD, PSD and PLD are always over-dispersed and PAD has advantage over PSD and PLD. The thunderstorms data have been considered in Tables 8-10.

Again it is obvious from fitting of PAD to thunderstorms data that PAD gives better fit than PD, PLD, and PSD in all data .

Table 4 Distribution of number of Chromatid aberrations ( $0.2 \mathrm{~g}$ chinon I, 24 hours)

\begin{tabular}{|c|c|c|c|c|c|}
\hline \multirow{2}{*}{$\begin{array}{l}\text { Number of } \\
\text { aberrations }\end{array}$} & \multirow{2}{*}{$\begin{array}{l}\text { Observed } \\
\text { frequency }\end{array}$} & \multicolumn{4}{|c|}{ Expected frequency } \\
\hline & & PD & PLD & PSD & PAD \\
\hline 0 & 268 & 231.3 & 257.0 & 257.6 & 259.0 \\
\hline I & 87 & 126.7 & 93.4 & 93.0 & 91.9 \\
\hline 2 & 26 & 34.7 & 32.8 & 32.7 & 32.5 \\
\hline 3 & 9 & 6.3 & 11.2 & 11.2 & 11.3 \\
\hline 4 & 4 & 0.8 & 3.8 & 3.7 & 3.8 \\
\hline 5 & 2 & $0.1\}$ & 1.2 & 1.2 & 1.3 \\
\hline , & 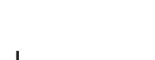 & 0.1 & 0.4 & 0.4 & 0.2 \\
\hline 6 & 1 & 0.1 & 0.2 & 0.2 & 0.0 \\
\hline $7+$ & 3 & & & & \\
\hline Total & 400 & 400.0 & 400.0 & 400.0 & 400.0 \\
\hline ML estimate & & $\hat{\theta}=0.5475$ & $\hat{\theta}=2.380442$ & $\hat{\theta}=2.829241$ & $\hat{\theta}=3.216733$ \\
\hline$\chi^{2}$ & & 38.21 & 6.21 & 6.28 & 6.5 \\
\hline d.f. & & 2 & 3 & 3 & 3 \\
\hline $\mathrm{p}$-value & & 0 & 0.1018 & 0.0987 & 0.0897 \\
\hline
\end{tabular}


Table 5 Mammalian cytogenetic dosimetry lesions in rabbit lymphoblast induced by streptonigrin (NSC-45383), Exposure $-60 \mu g \mid k g$

\begin{tabular}{|c|c|c|c|c|c|}
\hline \multirow{2}{*}{$\begin{array}{l}\text { Class/exposure } \\
(\mu g \mid k g)\end{array}$} & \multirow{2}{*}{$\begin{array}{l}\text { Observed } \\
\text { frequency }\end{array}$} & \multicolumn{4}{|c|}{ Expected frequency } \\
\hline & & PD & PLD & PSD & PAD \\
\hline 0 & 413 & 374.0 & 405.7 & 406.1 & 407.5 \\
\hline I & 124 & 177.4 & 133.6 & 132.9 & 131.2 \\
\hline 2 & 42 & 42.1 & 42.6 & 42.7 & 42.5 \\
\hline 3 & 15 & 6.6 & 13.3 & 13.4 & 13.6 \\
\hline 4 & 5 & 0.8 & $4.1)$ & 4.3 & 4.3 \\
\hline 5 & 0 & 0.1 & $1.2\}$ & $1.3\}$ & 1.3 \\
\hline 6 & 2 & 0.0 & 0.5 & 0.6 & 0.6 \\
\hline Total & 601 & 601.0 & 601.0 & 601.0 & 601.0 \\
\hline ML estimate & & $\hat{\theta}=0.47421$ & $\hat{\theta}=2.685373$ & $\hat{\theta}=3.492243$ & $\hat{\theta}=3.492243$ \\
\hline$\chi^{2}$ & & 48.17 & 1.34 & 1.10 & 0.70 \\
\hline d.f. & & 2 & 3 & 3 & 3 \\
\hline $\mathrm{p}$-value & & 0.0000 & 0.7196 & $0.777 \mid$ & 0.8732 \\
\hline
\end{tabular}

Table 6 Mammalian cytogenetic dosimetry lesions in rabbit lymphoblast induced by streptonigrin (NSC-45383), Exposure -70 $\mu g \mid \mathrm{kg}$

\begin{tabular}{|c|c|c|c|c|c|}
\hline \multirow{2}{*}{$\begin{array}{l}\text { Class/Exposure } \\
(\mu g \mid k g)\end{array}$} & \multirow{2}{*}{$\begin{array}{l}\text { Observed } \\
\text { frequency }\end{array}$} & \multicolumn{4}{|c|}{ Expected frequency } \\
\hline & & PD & PLD & PSD & PAD \\
\hline 0 & 200 & 172.5 & 191.8 & 192.0 & 192.8 \\
\hline I & 57 & 95.4 & 70.3 & 70.1 & 69.1 \\
\hline 2 & 30 & 26.4 & 24.9 & 24.9 & 24.8 \\
\hline 3 & 7 & 4.9 & 8.67 & 8.6 & $8.7)$ \\
\hline 4 & 4 & 0.7 & 2.9 & 2.9 & 3.0 \\
\hline 5 & 0 & 0.1 & 1.0 & 0.9 & 1.0 \\
\hline 6 & 2 & 0.0 & 0.5 & 0.6 & 0.6 \\
\hline Total & 300 & 300.0 & 300.0 & 300.0 & 300.0 \\
\hline ML estimate & & $\hat{\theta}=0.55333$ & $\hat{\theta}=2.353339$ & $\hat{\theta}=3.178185$ & $\hat{\theta}=3.178185$ \\
\hline$\chi^{2}$ & & 29.68 & 3.91 & 3.81 & 3.47 \\
\hline d.f. & & 2 & 2 & 2 & 2 \\
\hline $\mathrm{p}$-value & & 0.0000 & 0.1415 & 0.1488 & 0.1764 \\
\hline
\end{tabular}


Table 7 Mammalian cytogenetic dosimetry lesions in rabbit lymphoblast induced by streptonigrin (NSC-45383), Exposure -90 $\mu g \mid k g$

\begin{tabular}{|c|c|c|c|c|c|}
\hline \multirow{2}{*}{$\begin{array}{l}\text { Class/exposure } \\
(\mu g \mid k g)\end{array}$} & \multirow{2}{*}{$\begin{array}{l}\text { Observed } \\
\text { frequency }\end{array}$} & \multicolumn{4}{|c|}{ Expected frequency } \\
\hline & & PD & PLD & PSD & PAD \\
\hline 0 & 155 & 127.8 & 158.3 & 157.5 & 157.9 \\
\hline I & 83 & 109.0 & 77.2 & 77.5 & 76.8 \\
\hline 2 & 33 & 46.5 & 35.9 & 36.4 & 36.5 \\
\hline 3 & 14 & 13.2 & 16.1 & 16.4 & 16.6 \\
\hline 4 & 11 & 2.8 & $7.1)$ & $7.1)$ & $7.2)$ \\
\hline 5 & 3 & 0.5 & $3.1\}$ & $3.0\}$ & $3.0\}$ \\
\hline 6 & 1 & 0.2 & 2.3 & 2.1 & 2.0 \\
\hline Total & 300 & 300.0 & 300.0 & 300.0 & 300.0 \\
\hline ML estimate & & $\hat{\theta}=0.853333$ & $\hat{\theta}=2.034077$ & $\hat{\theta}=2.034077$ & $\hat{\theta}=2.431509$ \\
\hline$\chi^{2}$ & & 24.97 & 1.51 & $\mathrm{I} .74$ & 1.93 \\
\hline d.f. & & 2 & 3 & 3 & 3 \\
\hline $\mathrm{p}$-value & & 0 & 0.6799 & 0.6281 & $0.587 \mid$ \\
\hline
\end{tabular}

Table 8 Observed and expected number of days that experienced $X$ thunderstorms events at Cape Kennedy, Florida for the II-year period of record for the month of June, January 1957 to December 1967, Falls et al., ${ }^{24}$

\begin{tabular}{|c|c|c|c|c|c|}
\hline \multirow{2}{*}{$\begin{array}{l}\text { No. of } \\
\text { thunderstorms }\end{array}$} & \multirow{2}{*}{$\begin{array}{l}\text { Observed } \\
\text { frequency }\end{array}$} & \multicolumn{4}{|c|}{ Expected frequency } \\
\hline & & PD & PLD & PSD & PAD \\
\hline 0 & 187 & 155.6 & 185.3 & 184.8 & 185.4 \\
\hline I & 77 & 117.0 & 83.5 & 83.6 & 82.7 \\
\hline 2 & 40 & 43.9 & 35.9 & 36.3 & 36.3 \\
\hline 3 & 17 & 11.0 & 15.0 & 15.2 & 15.4 \\
\hline 4 & 6 & 2.1 & $6.1)$ & $6.1)$ & 6.3 \\
\hline 5 & 2 & 0.3 & 2.5 & $2.4\}$ & $2.4\}$ \\
\hline 6 & 1 & 0.1 & 1.7 & 1.6 & 1.5 \\
\hline Total & 330 & 330.0 & 330.0 & 330.0 & 330.0 \\
\hline ML estimate & & $\hat{\theta}=0.751515$ & $\hat{\theta}=1.804268$ & $\hat{\theta}=2.625345$ & $\hat{\theta}=2.625345$ \\
\hline$\chi^{2}$ & & 31.93 & 1.43 & 1.25 & 1.07 \\
\hline d.f. & & 2 & 3 & 3 & 3 \\
\hline $\mathrm{p}$-value & & 0.0000 & 0.6985 & $0.74 I$ & 0.7843 \\
\hline
\end{tabular}


Table 9 Observed and expected number of days that experienced $X$ thunderstorms events at Cape Kennedy, Florida for the II-year period of record for the month of July, January 1957 to December 1967, Falls et al., ${ }^{24}$

\begin{tabular}{|c|c|c|c|c|c|}
\hline \multirow{2}{*}{$\begin{array}{l}\text { No. of } \\
\text { thunderstorms }\end{array}$} & \multirow{2}{*}{$\begin{array}{l}\text { Observed } \\
\text { frequency }\end{array}$} & \multicolumn{4}{|c|}{ Expected frequency } \\
\hline & & PD & PLD & PSD & PAD \\
\hline 0 & 177 & 142.3 & 177.7 & 176.5 & 176.7 \\
\hline I & 80 & 124.4 & 88.0 & 88.4 & 87.6 \\
\hline 2 & 47 & 54.3 & 41.5 & 42.2 & 42.3 \\
\hline 3 & 26 & 15.8 & 18.9 & 19.2 & 19.5 \\
\hline 4 & 9 & \multirow{2}{*}{$\left.\begin{array}{l}3.5 \\
0.7\end{array}\right\}$} & $8.4\}$ & $8.5\}$ & 8.6 \\
\hline 5 & 2 & & $6.5\}$ & $6.2\}$ & $6.3\}$ \\
\hline Total & 341 & 341.0 & 341.0 & 341.0 & 341.0 \\
\hline ML estimate & & $\hat{\theta}=0.873900$ & $\hat{\theta}=1.583536$ & $\hat{\theta}=1.995806$ & $\hat{\theta}=2.390474$ \\
\hline$\chi^{2}$ & & 39.74 & 5.15 & 4.67 & 4.35 \\
\hline d.f. & & 2 & 3 & 3 & 3 \\
\hline p-value & & 0.0000 & 0.1611 & 0.1976 & 0.2261 \\
\hline
\end{tabular}

Table 10 Observed and expected number of days that experienced $\mathrm{X}$ thunderstorms events at Cape Kennedy, Florida for the $\mathrm{I} \mathrm{I}$-year period of record for the month of August, January 1957 to December 1967, Falls et al., ${ }^{24}$

\begin{tabular}{|c|c|c|c|c|c|}
\hline \multirow{2}{*}{$\begin{array}{l}\text { No. of } \\
\text { thunderstorms }\end{array}$} & \multirow{2}{*}{$\begin{array}{l}\text { Observed } \\
\text { frequency }\end{array}$} & \multicolumn{4}{|c|}{ Expected frequency } \\
\hline & & PD & PLD & PSD & PAD \\
\hline 0 & 185 & 151.8 & 184.8 & 184.1 & 184.7 \\
\hline I & 89 & 122.9 & 87.2 & 87.5 & 86.6 \\
\hline 2 & 30 & 49.7 & 39.3 & 39.8 & 39.8 \\
\hline 3 & 24 & 13.4 & 17.1 & 17.3 & 17.6 \\
\hline 4 & 10 & $2.7\}$ & 7.3 & 7.4 & 7.4 \\
\hline 5 & 3 & 0.5 & $5.3\}$ & $4.9\}$ & $4.9\}$ \\
\hline Total & 341 & 341.0 & 341.0 & 341.0 & 341.0 \\
\hline ML estimate & & $\hat{\theta}=0.809384$ & $\hat{\theta}=0.809384$ & $\hat{\theta}=2.114545$ & $\hat{\theta}=2.511962$ \\
\hline$\chi^{2}$ & & 49.49 & 5.03 & 5.06 & 4.83 \\
\hline d.f. & & 2 & 3 & 3 & 3 \\
\hline p-value & & 0.0000 & 0.1696 & 0.1674 & 0.1847 \\
\hline
\end{tabular}

\section{Acknowledgement}

None.

\section{Conflict of interest}

None.

\section{References}

1. Shanker R. The discrete Poisson-Amarendra. International Journal of Statistical Distributions and Applications. 2016b.

2. Sankaran M. The discrete Poisson-Lindley distribution. Biometrics. 1970;26(1):145-149. 
3. Shanker R. The discrete Poisson-Sujatha distribution. International Journal of Probability and Statistics. 2016d;5(1):1-9.

4. Shanker R. Amarendra distribution and Its Applications. American Journal of Mathematics and Statistics. 2016a;6(1):44-56.

5. Shanker R. Shanker distribution and Its Applications. International Journal of Statistics and Applications. 2015a;5(6):338-348.

6. Shanker R. Akash distribution and Its Applications. International Journal of Probability and Statistics. 2015b;4(3):65-75.

7. Shanker R. Sujatha distribution and Its Applications. Statistics in Transition new Series. 2016c;17(3).

8. Lindley DV. Fiducial distributions and Bayes theorem. Journal of Royal Statistical Society, Series. 1958;B20(1):102-107.

9. Shanker R, Hagos F. On Poisson-Lindley distribution and Its applications to Biological Sciences. Biometrics \& Biostatistics International Journal. 2015;2(4):1-5.

10. Shanker R, Hagos F, Sujatha S, et al. On zero-truncation of Poisson and Poisson-Lindley distributions and Their Applications. Biometrics \& Biostatistics International Journal. 2015;2(6):1-14.

11. Shanker R, Hagos F. On Poisson-Sujatha distribution and Its Applications to model count data from biological sciences. Biometrics \& Biostatistics International Journal. 2016d;3(4):1-7.

12. Shanker R, Hagos F. Size-biased Poisson-Sujatha distribution with Applications. American Journal of Mathematics and Statistics. 2016a;6(4):145-154.

13. Shanker R, Hagos F. Zero-truncated Poisson-Sujatha distribution with Applications. Journal of Ethiopian Statistical Association. 2016b.

14. Shanker R, Hagos F. On zero-truncation of Poisson, Poisson-Lindley, and Poisson-Sujatha distribution and their Applications. Biometrics \& Biostatistics International Journal. 2016c;3(5):1-13.
15. Johnson NL, Kotz S, Kemp AW. Univariate Discrete Distributions. $2^{\text {nd }}$ edn, John Wiley \& sons Inc, USA. 1992.

16. Fisher RA, Corpet AS, Williams CB. The relation between the number of species and the number of individuals in a random sample of an animal population. Journal of Animal Ecology. 1943;12(1):42-58.

17. Kempton RA. A generalized form of Fisher's logarithmic series. Biometrika. 1975;62(1):29-38.

18. Tripathi RC, Gupta RC. A generalization of the $\log$-series distribution. Comm in Stat (Theory and Methods). 1985;14(8):1779-1799.

19. Mishra A, Shanker R. Generalized logarithmic series distributionIts nature and applications. Proceedings of the Vth International Symposium on Optimization and Statistics. 2002;155-168.

20. Loeschke V, Kohler W. Deterministic and Stochastic models of the negative binomial distribution and the analysis of chromosomal aberrations in human leukocytes. Biometrical Journal. 1976;18(6):427-451.

21. Janardan KG, Schaeffer DJ. Models for the analysis of chromosomal aberrations in human leukocytes. Biometrical Journal. 1977;19 (8):599-612.

22. Catcheside DG, Lea DE, Thoday JM. Types of chromosome structural change induced by the irradiation on Tradescantia microspores. $J$ Genet. 1946;47:113-136.

23. Mc Guire JU, Brindley TA, Bancroft TA. The distribution of European corn-borer larvae pyrausta in field corn. Biometrics. 1957;13(1):65-78.

24. Catcheside DG, Lea DE, Thoday JM. The production of chromosome structural changes in Tradescantia microspores in relation to dosage, intensity and temperature. J Genet. 1946;47:137-149. 